\title{
New Results on Diffractive and Exclusive Production from CDF
}

\section{Konstantin GOULIANOS**}

The Rockefeller University

E-mail: dino@rockefeller.edu

We present results on central exclusive production of $\pi^{+} \pi^{-}$in p p collisions at $\sqrt{s}=900$ and $1960 \mathrm{GeV}$ using events with two charged hadrons in the final state within the pseudorapidity region $|\eta| \leq 1.3$ and no particles in $|\eta|>1.3$. These results open a new window into hadron spectroscopy, and may be used as benchmarks for testing relevant theoretical models.

XXI International Workshop on Deep-Inelastic Scattering and Related Subject-DIS2013,

22-26 April 2013

Marseilles,France

\footnotetext{
* Speaker.

† Presented on behalf of the CDF Collaboration.
} 


\section{Introduction}

The CDF Collaboration (CDF) has been studying diffraction in $\bar{p} p$ collisions for the past quarter century, aiming to understand the QCD aspects of the diffractive exchange, a strongly interacting color-singlet quark/gluon combination with vacuum quantum numbers, traditionally referred to as Pomeron $(\mathbb{P})$ exchange. Such exchanges lead to large, non-exponentially suppressed pseudorapidity regions devoid of particles, called rapidity ${ }^{1}$ gaps. Diffractive processes are classified as single dissociation or single diffraction, $\mathrm{SD}$, characterized by a forward gap adjacent to a surviving $\bar{p}$ or $p$, double dissociation or double diffraction, $\mathrm{DD}$, characterized by a central gap, and central diffraction or double-Pomeron exchange, CD or DPE, a process with two forward gaps.

A special class of diffraction is central exclusive production, a DPE process in which a specific state is centrally produced [1]. CDF has published results on exclusive dijet (2008) [2], $\mu^{+} \mu^{-}: \chi_{c}$, $J / \psi$ and $J / \psi(2 s)$ (2009) [3], and $\gamma \gamma / e^{+} e^{-}$(2012) [4] production. Here, we report on the observation of exclusive $\pi^{+} \pi^{-}$production [5] and compare the results with theoretical expectations.

\section{Central exclusive production of $\pi^{+} \pi^{-}$}

\subsection{Detector, triggers, datasets}

Detector. The CDF II detector is shown schematically in Fig 1. It consists of the main detector, labeled CDF-II in this figure, equipped with a tracking system and calorimeters (central: CCAL, plug: PCAL), and the forward components (Cherenkov Luminosity Counters: CLC, MiniPlugs: MP, Roman Pot Spectrometer: RPS). The RPS and MPs were not active in this study, and from the BSCs only those covering the pseudorapidity region of $5.4<|\eta|<5.9$ are used.

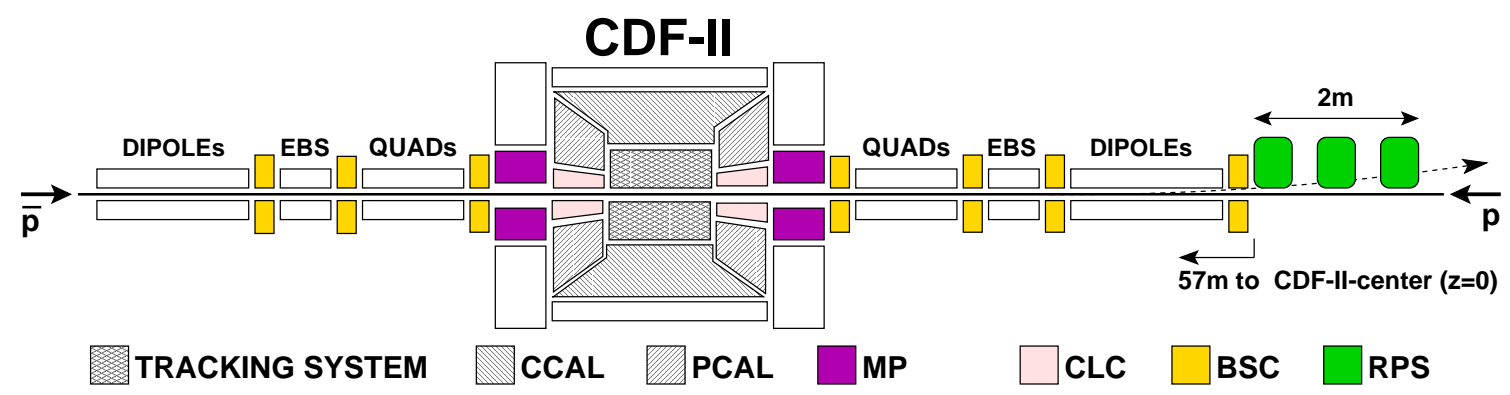

Figure 1: Schematic plan view of the CDF II detector showing the tracking system and calorimeters (CCAL, PCAL), and forward components (MP, CLC, BSC, RPS); EBSs are electrostatic beam separators.

Triggers. The following two triggeres were used for data collection:

- signal: two CCAL towers $(|\eta|<1.3)$ with energy $\mathrm{E}>0.5 \mathrm{GeV}$ (a very low threshold!) and no energy in BSC $(|\eta|=5.4-5.9)$ and in the Forward Plug Calorimeters $(|\eta|=2.11-3.64)$.

- zero-bias: offline selected bunch-crossing events with no tracks for noise/exclusivity studies.

Datasets. The signal datasets consist of $90(22) \times 10^{6}$ events at $\sqrt{s}=1960(900) \mathrm{GeV}$.

\footnotetext{
${ }^{1}$ Rapidity, $y=\frac{1}{2} \ln \frac{E+p_{L}}{E-p_{L}}$, and pseudorapidity, $\eta=-\ln \tan \frac{\theta}{2}$, where $\theta$ is the polar angle of a particle w.r.t. the proton beam $(+\hat{z})$, are used interchangeably for particles detected in the calorimeters, as they are approximately equal.
} 


\subsection{Preliminary results}

We report results for events with exactly two tracks within rapidity $\left|y_{\pi^{+}} \pi^{-}\right|<1.0$ and $\mathrm{M}_{\pi^{+} \pi^{-}}<$ 0.8 , where there is useful acceptance at all $\mathrm{p}_{T}$. No particle ID is (yet) being used, and the observed tracks are assumed to be due to pions.

We select events in regions of instantaneous luminosity $1 \times 10^{30}<L<2.2 \times 10^{30} \mathrm{~cm}^{-2} \mathrm{~s}^{-1}$ (Fig. 2-left), and set detector thresholds for optimum signal/noise ratio (Fig. 2-right).
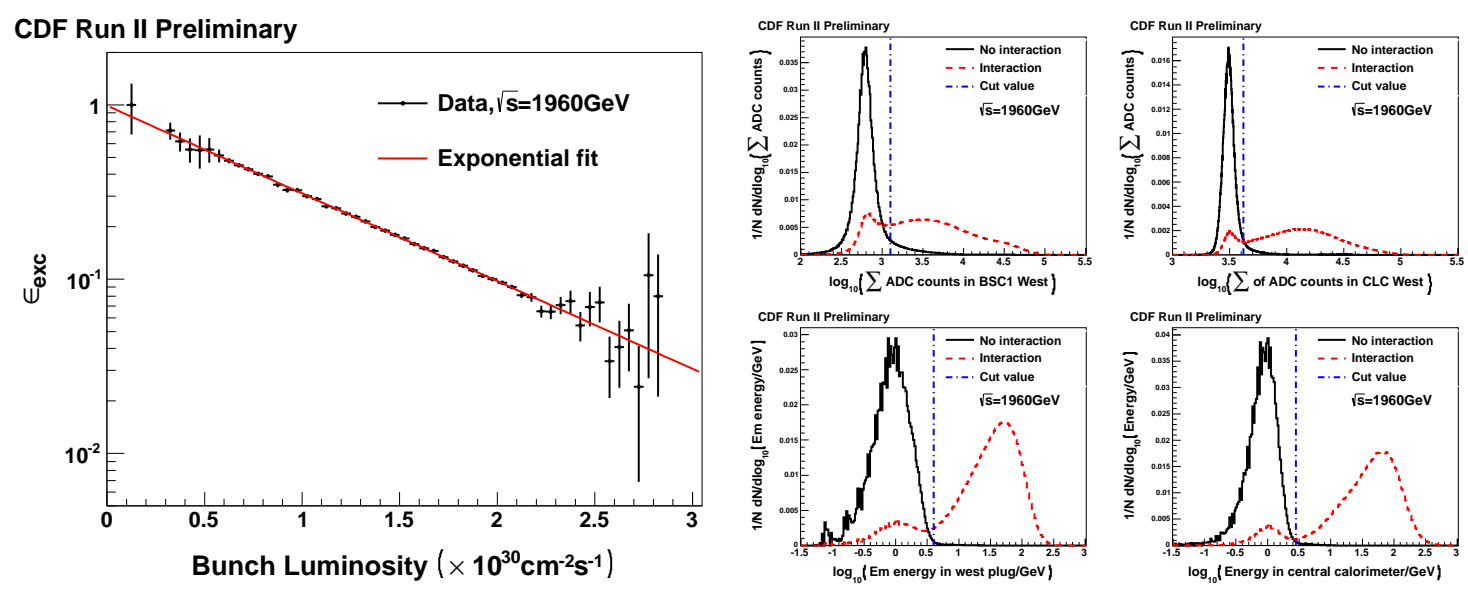

Figure 2: Zero-bias data sample at $\sqrt{s}=1960 \mathrm{GeV}$ with an exponential fit: (left) efficiency of event selection (probability that the whole detector is empty) vs beam-bunch instantaneous luminosity for a single bunch (the $L$ quoted in the text is 36 times larger, as there are 36 colliding bunches); (right) detector-noise levels for "interaction" and "no-interaction" events. The vertical dashed lines show the cuts used to define "empty" detectors, or "noise" (the $K^{+} K^{-}$background in this area, measured with $K^{0} K^{0}$ events, amounts to only a few \%).

Figure 3 shows mass distributions of $\pi^{+} \pi^{-}$candidate events uncorrected (left) and corrected (right) for acceptance. The $f_{0}(980), f_{2}(1270)$, and $f_{0}(1370)$ are clearly visible. The small but significant peak at $3.1 \mathrm{GeV}$ is understood to be from $J / \psi \rightarrow e^{+} e^{-}$with $\mathrm{M}_{e^{+} e^{-}}$treated as $\mathrm{M}_{\pi^{+} \pi^{-}}$.
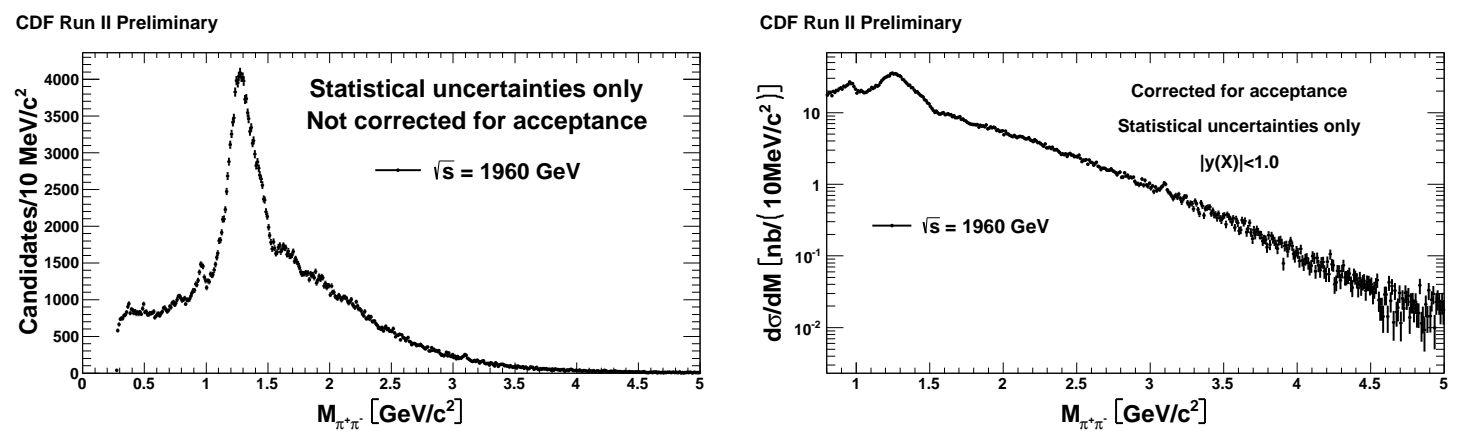

Figure 3: $\mathrm{M}_{\pi^{+} \pi^{-}}$distributions at $\sqrt{s}=1960 \mathrm{GeV}$ not corrected (left) and corrected (right) for acceptance.

Figure 4 shows the ratio of $\pi^{+} \pi^{-}$candidates at $\sqrt{s}=1960 / 900 \mathrm{GeV}$ (top), and the mean $\mathrm{p}_{t}$ for $\sqrt{s}=1960 \mathrm{GeV}$ (bottom-left) and $\sqrt{s}=900 \mathrm{GeV}$ (bottom-right) vs $\mathrm{M}_{\pi^{+}} \pi^{-}$. The statistically 
more significant data at $\sqrt{s}=1960 \mathrm{GeV}$ show structures at $1.5 \mathrm{GeV}, 2.25 \mathrm{GeV}$, and between $3 \mathrm{GeV}$ and $4 \mathrm{GeV}$. Work is in progress to understand these structures, including a phase-shift analysis.

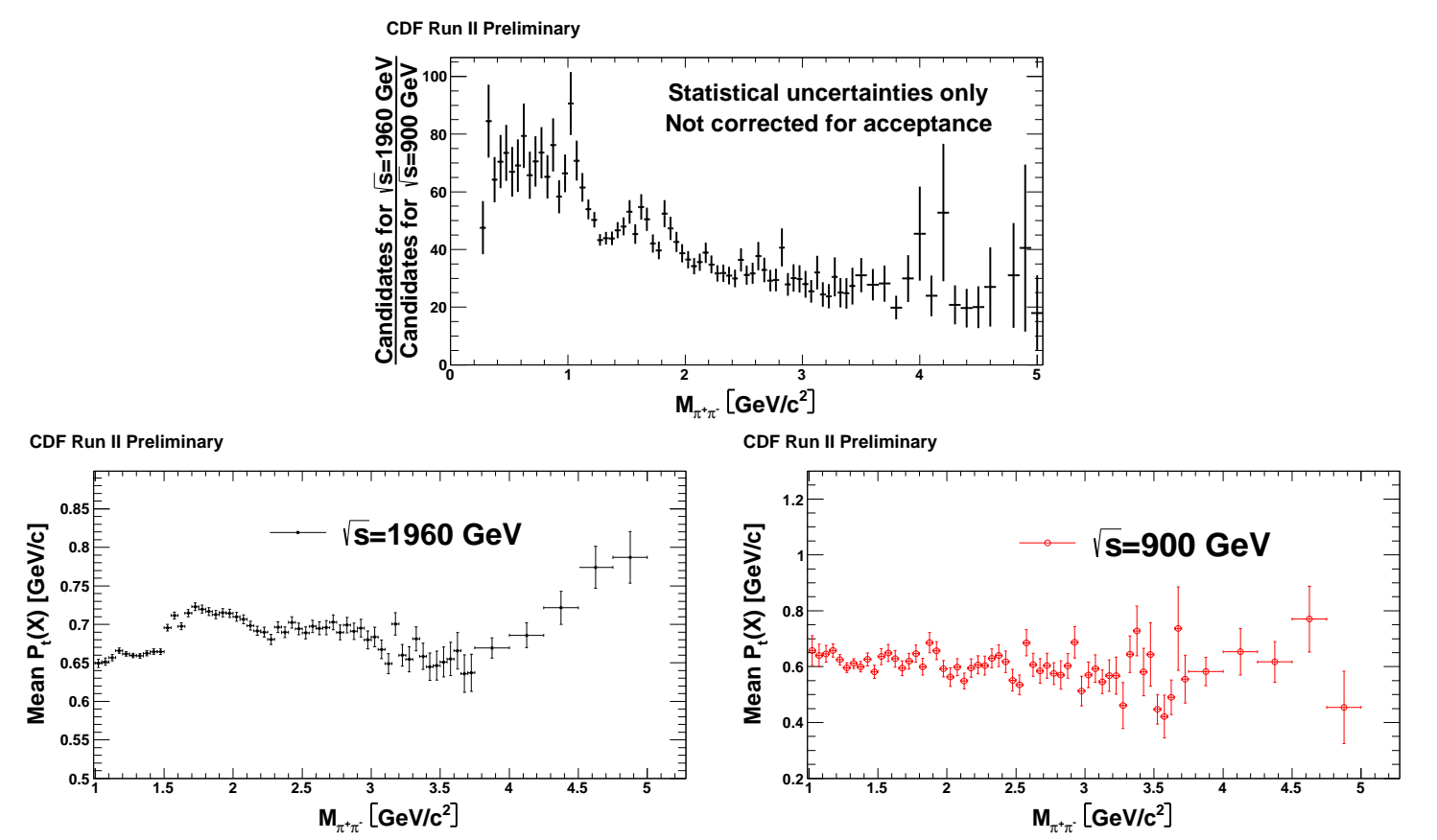

Figure 4: Ratio of events at $\sqrt{s}=1960 / 900 \mathrm{GeV}$ (top), and mean $\mathrm{p}_{t}$ of $\pi^{+} / \pi^{-}$(left/right) vs $\mathrm{M}_{\pi^{+}} \pi^{-}$.

In Fig. 5, we compare the distributions of $d \sigma / d \mathrm{M}_{\pi^{+} \pi^{-}}$of events at $\sqrt{s}=1960$ and $900 \mathrm{GeV}$ for $\mathrm{M}_{\pi^{+} \pi^{-}}<5 \mathrm{GeV}$ (left), and zoom into the region of $\mathrm{M}_{\pi^{+} \pi^{-}}<2 \mathrm{GeV}$ (right) for an expanded view. At $\mathrm{M}_{\pi^{+} \pi^{-}}>1.5 \mathrm{GeV}$, we observe features in the mass spectrum which are not yet understood and are the subject of further studies currently underway, including a partial wave analysis.
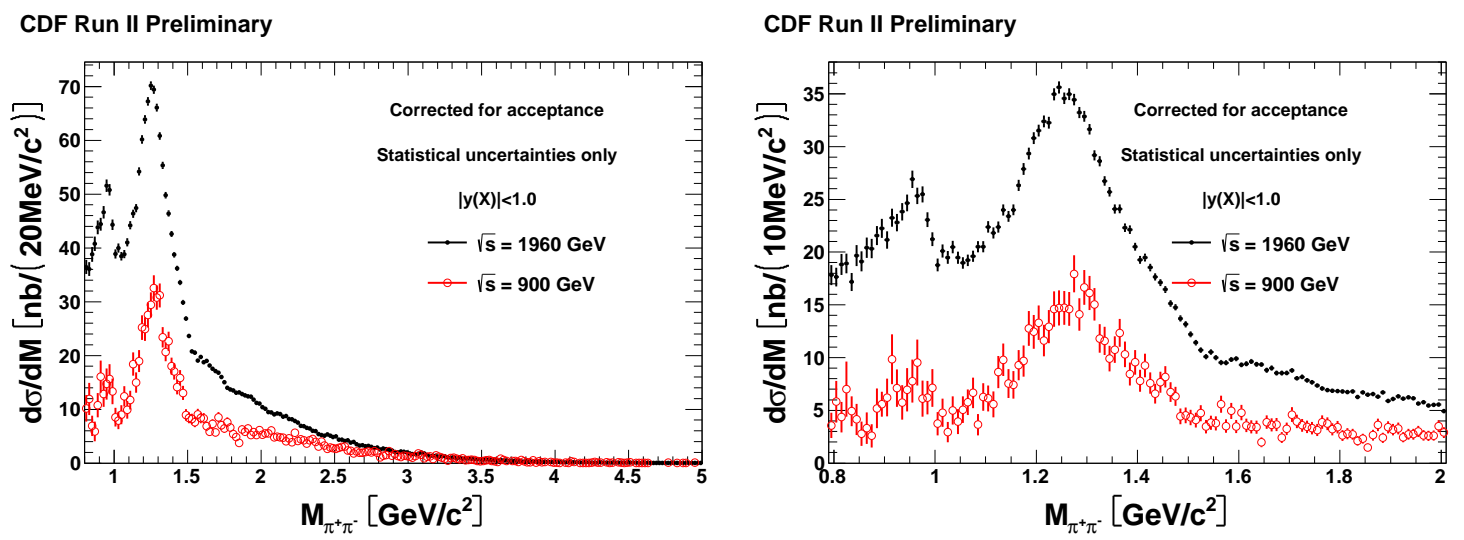

Figure 5: Differential cross sections $d \sigma / d \mathrm{M}_{\pi^{+} \pi^{-}}$vs $\mathrm{M}_{\pi^{+} \pi^{-}}$at $\sqrt{s}=1960$ and $900 \mathrm{GeV}$ for the mass regions $\mathrm{M}_{\pi^{+} \pi^{-}}<5 \mathrm{GeV}$ (left) and $\mathrm{M}_{\pi^{+} \pi^{-}}<2 \mathrm{GeV}$ (right). 


\section{Summary}

We have measured exclusive $\pi^{+} \pi^{-}$production in $\bar{p} p$ collisions at $\sqrt{s}=900 \mathrm{GeV}$ and $\sqrt{s}=$ $1960 \mathrm{GeV}$ with the CDF II detector at the Fermilab Tevatron Collider. Using tracks, assumed to be from pions which are the dominant charged-pair component, we explored the low mass region of $\mathrm{M}_{\pi^{+} \pi^{-}}<5 \mathrm{GeV}$. We observe the well known resonances $f_{0}(980)$ and $f_{2}(1270)$, and see a small but significant peak at $3.1 \mathrm{GeV}$ understood to be from $J / \psi \rightarrow e^{+} e^{-}$with $\mathrm{M}_{e^{+} e^{-}}$assumed as $\mathrm{M}_{\pi^{+} \pi^{-}}$. We also observe features at $\mathrm{M}_{\pi^{+} \pi^{-}}>1.5 \mathrm{GeV}$ which are not yet understood. Further investigations of these features and a partial wave analysis are currently underway.

\section{Acknowledgements}

I would like to thank my colleagues at the CDF Collaboration who made this work possible, and the Office of Science of the Department of Energy for financial support.

\section{References}

[1] M. G. Albrow, T. D. Coughlin and J. R. Forshaw, Central Exclusive Particle Production at High Energy Hadron Colliders, Prog. Part. Nucl. Phys. 65, 149 (2010) [hep-ph/1006.1289].

[2] T. Aaltonen et al. (CDF Collaboration), Observation of Exclusive Dijet Production at the Fermilab Tevatron $\bar{p} p$ Collider, Phys. Rev. D 77, 052004 (2008) [hep-ex/1206.3995].

[3] T. Aaltonen et al. (CDF Collaboration), Observation of Exclusive Charmonium Production and $\gamma \gamma \rightarrow \mu^{+} \mu^{-}$in $\bar{p} p$ Collisions at $\sqrt{s}=1.96 \mathrm{TeV}$, Phys. Rev. Lett. 102, 242001 (2009) [hep-ex/0902.1271].

[4] T. Aaltonen et al. (CDF Collaboration), Observation of Exclusive $\gamma \gamma$ Production in p $\bar{p}$ Collisions at $\sqrt{s}=1.96 \mathrm{TeV}$, Phys. Rev. Lett. 108, 081801 (2012) [hep-ex/1112.0858].

[5] M. G. Albrow (Representing the CDF Collaboration), Central Exclusive Production of Hadrons in CDF, in proceedings of DIFFRACTION 2012: International Workshop on Diffraction in High Energy Physics, 10-15 September 2012, AIP Conf. Proc. 1523, pp. 294-297 (2013) [doi:http://dx.doi.org/10.1063/1.4802170]. 